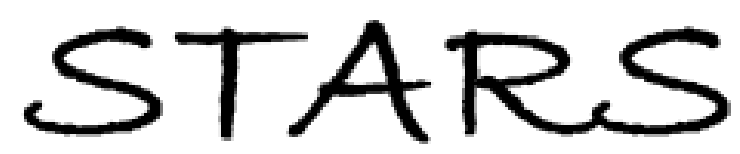

University of Central Florida

STARS

Faculty Bibliography 2010s

Faculty Bibliography

$1-1-2014$

\title{
Non-ideal optical isotropy of blue phase liquid crystal
}

Yi-Fen Lan

University of Central Florida

Yifan Liu

University of Central Florida

Pu-Jung Huang

Daming Xu

University of Central Florida

Cheng-Yeh Tsai

See next page for additional authors

Find similar works at: https://stars.library.ucf.edu/facultybib2010

University of Central Florida Libraries http://library.ucf.edu

This Article is brought to you for free and open access by the Faculty Bibliography at STARS. It has been accepted for inclusion in Faculty Bibliography 2010 s by an authorized administrator of STARS. For more information, please contactSTARS@ucf.edu.

\section{Recommended Citation}

Lan, Yi-Fen; Liu, Yifan; Huang, Pu-Jung; Xu, Daming; Tsai, Cheng-Yeh; Lin, Chin-Huan; Sugiura, Norio; and Wu, Shin-Tson, "Non-ideal optical isotropy of blue phase liquid crystal" (2014). Faculty Bibliography 2010s. 5615.

https://stars.library.ucf.edu/facultybib2010/5615

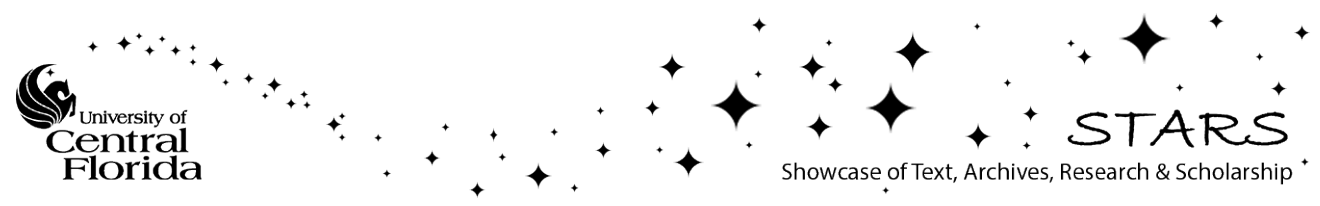


Authors

Yi-Fen Lan, Yifan Liu, Pu-Jung Huang, Daming Xu, Cheng-Yeh Tsai, Chin-Huan Lin, Norio Sugiura, and ShinTson Wu 


\section{Non-ideal optical isotropy of blue phase liquid crystal}

Cite as: Appl. Phys. Lett. 105, 011903 (2014); https://doi.org/10.1063/1.4887371

Submitted: 29 April 2014 . Accepted: 25 June 2014 . Published Online: 08 July 2014

Yi-Fen Lan, Yifan Liu, Pu-Jung Huang, Daming Xu, Cheng-Yeh Tsai, Chin-Haun Lin, Norio Sugiura, and Shin-Tson Wu
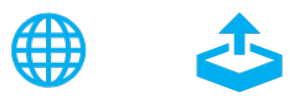

\section{ARTICLES YOU MAY BE INTERESTED IN}

Electro-optics of polymer-stabilized blue phase liquid crystal displays

Applied Physics Letters 94, 101104 (2009); https://doi.org/10.1063/1.3097355

Electro-optic response of polymer-stabilized blue phase liquid crystals

Applied Physics Letters 105, 011119 (2014); https://doi.org/10.1063/1.4890031

A full-color reflective display using polymer-stabilized blue phase liquid crystal

Applied Physics Letters 102, 081102 (2013); https://doi.org/10.1063/1.4793750

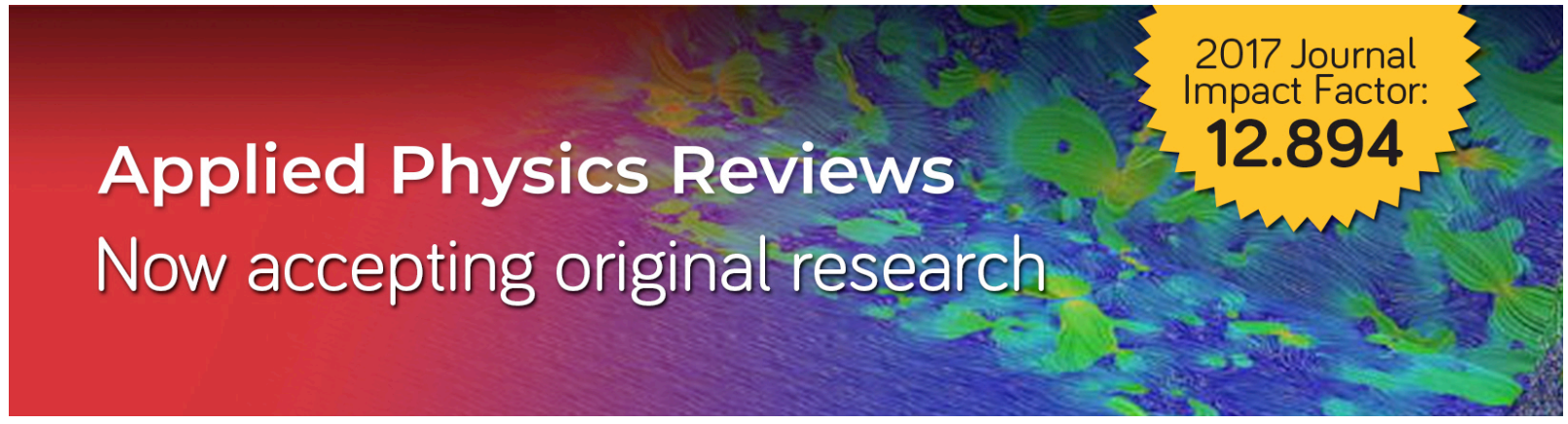




\title{
Non-ideal optical isotropy of blue phase liquid crystal
}

\author{
Yi-Fen Lan, ${ }^{1,2, a)}$ Yifan Liu, ${ }^{2}$ Pu-Jung Huang, ${ }^{1}$ Daming Xu, ${ }^{2}$ Cheng-Yeh Tsai, ${ }^{1}$ \\ Chin-Haun Lin, ${ }^{1}$ Norio Sugiura, ${ }^{1}$ and Shin-Tson $\mathrm{Wu}^{2}$ \\ ${ }^{1}$ Advanced Display Mode \& Material Research Center, AU Optronics Corporation, Hsinchu 30078, Taiwan \\ ${ }^{2}$ The College of Optics \& Photonics, University of Central Florida, Orlando, Florida 32816, USA
}

(Received 29 April 2014; accepted 25 June 2014; published online 8 July 2014)

\begin{abstract}
Since 1981, blue phase liquid crystal (BPLC) was regarded as optical isotropy and high contrast ratio (CR) should be achieved easily. However, low CR of BPLC display was reported in all literatures. Here, we show BPLC is non-ideal optical isotropy which leads to poor CR. In our report, BPLC not only revealed primary structure of double-twist cylinder and secondary structure of lattice but also revealed tertiary structure of self-alignment on electrode surface. This finding will be useful to improve CR and inspire researches in display industry and academics. (C) 2014 AIP Publishing LLC. [http://dx.doi.org/10.1063/1.4887371]
\end{abstract}

Polymer-stabilized blue phase liquid crystal (BPLC) was well defined as three-dimension nanostructures and revealed three thermodynamic lattices, blue phase I, blue phase II, and blue phase III. ${ }^{1,2}$ Nanostructure of BPLC showed excellent optoelectric properties. For example, optical isotropy showed alignment free and voltage-off state. Short coherent length contributed to microsecond response time. ${ }^{3,4}$ Consideration above advantages; BPLC was regarded as next-generation display. Initially, BPLC display rendered many issues such as narrow stable temperature range, high driving voltage, large hysteresis, and poor charging. However, all of these issues have been overcome. Stable temperature range of BPLC was enlarged by polymer stabilization. ${ }^{5}$ Low driving voltage of $8.4 \mathrm{~V}_{\mathrm{rms}}$ was achieved by electrode design and BPLC materials. ${ }^{6,7}$ Hysteresis free was obtained by vertical-field switching (VFS) and dual-frequency driving. ${ }^{8,9}$ Full charge was proposed by bootstrapping method. ${ }^{10}$ In 2013, BPLC display was also demonstrated by using groove cell. ${ }^{11}$

Since 1981, BPLC was regarded as optical isotropic material and high contrast ratio (CR) should be achieved easily. However, low CR was reported in all literatures. ${ }^{12-14}$ In this report, BPLC reveals non-ideal optical isotropy which leads to low CR. In order to investigate issue of optical isotropy, dark state of BPLC was analyzed by laser-detector system. ${ }^{14}$ R633 nm was used for light source of laser. Transmission axis of the front linear polarizer was fixed and crossed to the analyzer. BPLC sample was located between polarizer and analyzer. Light leakage was collected and measured by laser detector. Without a sample, the extinction ratio of the crossed polarizers exceeds $10^{5}: 1$. During the measurement of dark state, test cell was rotated from $0^{\circ}$ to $90^{\circ}$, while maintained polarizer crossed to analyzer. In other word, electrode axis of in-plane-switching (IPS) cell was changed corresponding to transmitted axis of analyzer. As showed in Figure 1(a), $\theta$ was defined as angle between electrode axis of IPS cell and transmitted axis of analyzer. For example, if electrode axis of IPS was parallel to transmitted axis of analyzer, $\theta$ was $0^{\circ}$. If $\theta$ showed in $45^{\circ}$, electrode axis of IPS was cross to transmitted axis of analyzer in $45^{\circ}$. If electrode axis

\footnotetext{
${ }^{\text {a) }}$ Author to whom correspondence should be addressed. Electronic mail: yifenlan@ntu.edu.tw
}

of IPS was perpendicular to transmitted axis of analyzer, $\theta$ was $90^{\circ}$.

In our experiment, BPLC revealed optical spectrum of ultraviolet range and the dark state was measured at $\theta=0^{\circ}-90^{\circ}$. Initially, dark state of empty cell was analyzed and showed in Figure 1(b). Both IPS and VFS cell showed small dark state $\left(<1.00 \times 10^{-3} \%\right)$ and revealed no significant change at different $\theta$. Subsequently, BPLC was injected into VFS cell. After polymeric stabilization, dark state of VFS revealed the same trend of empty cell (Figure 1(c)). It showed no significant change at different $\theta$, but dark state revealed one order larger $\left(3.33 \times 10^{-2} \%\right)$ than empty cell. However, BPLC revealed different manner in dark state of IPS cell (Figure 1(d)). Result showed large dark state of $1.20 \times 10^{-2} \%$ at $\theta=45^{\circ}$ and small dark state of $1.07 \times 10^{-2} \%$ and $1.01 \times 10^{-2} \%$ at $\theta=0^{\circ}$ and $\theta=90^{\circ}$, respectively. Compared to VFS with IPS cell, light leakage of VFS cell was three-times larger than that of IPS cell. Overall, dark state revealed trend of VFS $>$ IPS $>$ empty cell. According to dark state result of IPS cell, BPLC can be regarded as non-ideal optical isotropic material. For example, if BPLC was perfect optical isotropic material, dark state should be cell-structure independence and showed high CR. However, experimental data revealed different dark state in IPS cell at variation of $\theta$. Consequently, BPLC was regarded as non-ideal optical material which can be explained by twist-nematic liquid crystal (TNLC) model. ${ }^{14}$

Consideration to interaction between electrode and BPLC, mechanism of BPLC self-assembly and alignment were proposed (Figure 2). Ideally, BPLC was regarded as optical isotropy and linear polarization light showed the same optical behavior after passed through BPLC (Figure 2(a)). However, it revealed different manner in practical research. Generally, it was well known that TNLC display rendered normally white and incident light passed through optoelectric device at voltage-off state. BPLC revealed similar anti-isotropic behavior as TNLC, but only performed in nanodomain. As showed in Figure 2(b), non-ideal optical isotropic BPLC was consisted by BPLC lattices which rendered dimension in nanoscale. ${ }^{9}$ In other word, when incident light passed through nanoscale lattice of non-ideal optical isotropic BPLC, phase retardation was found due to non- 
(a)
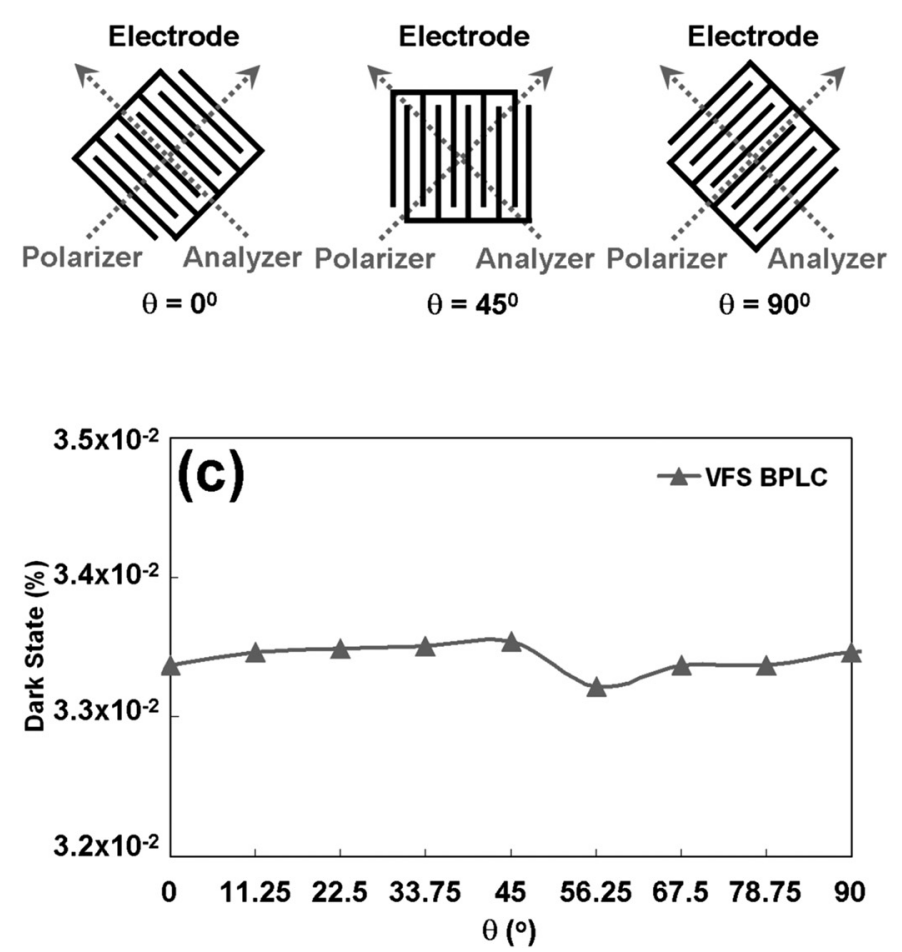
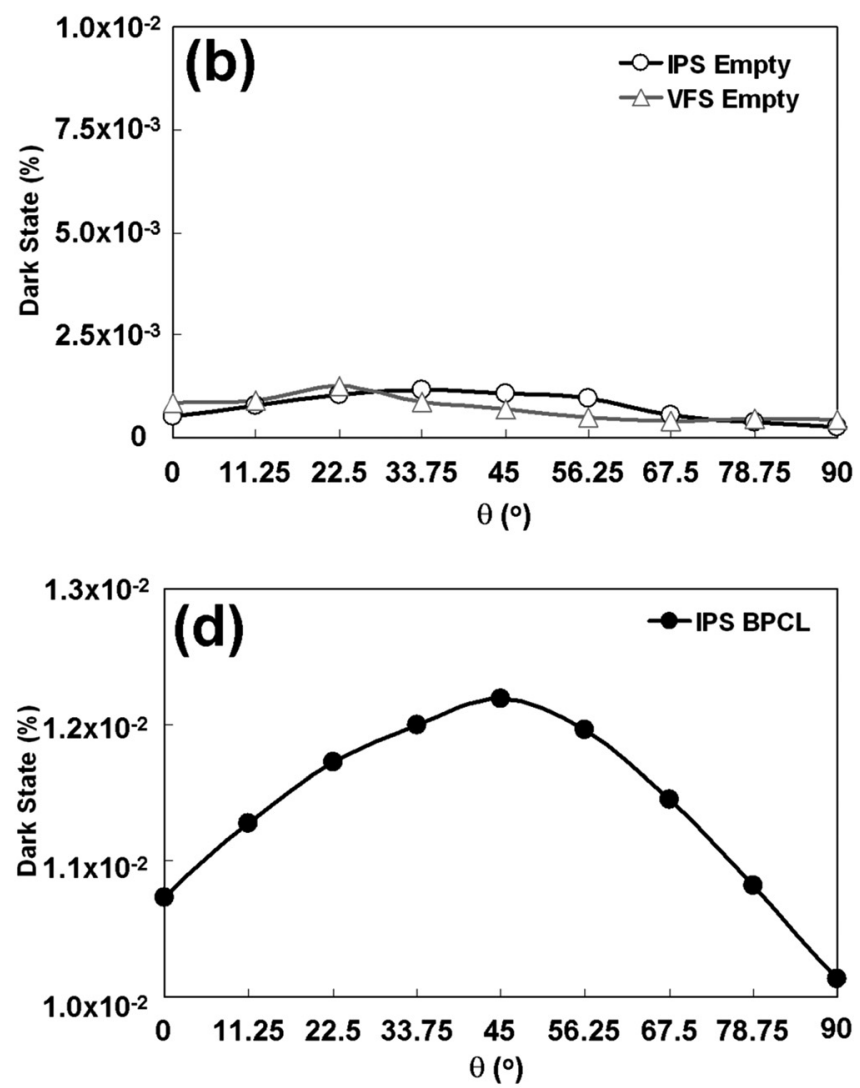

FIG. 1. Measurement of dark state by laser-detector system: (a) Definition of angle between transmittance axis of analyzer and electrode axis; (b) dark state of empty cell; (c) dark state of VFS BPLC cell, and (d) dark state of IPS BPLC cell.

ideal optical isotropy of BPLC. This indicated that linear polarization light revealed slightly rotated after incident light passed through BPLC (orange line). The rotation direction was correlated to the rotatory power of BPLC. For example, if BPLC revealed right-hand rotatory power, the incident light showed in slightly right-hand rotation.

In addition, TNLC showed alignment in electrode surface due to anchoring force between TNLC and electrode. BPLC also revealed similar alignment behavior as TNLC, but only performed in nanoscale. As showed in Figure 2(c),
BPLC lattices interacted with electrode surface and revealed self-assembly into elliptical tertiary structures. It was well known that BPLC lattice was consisted by primary structure of double twist cylinder (DTC) which revealed dimension in diameter of $10 \mathrm{~nm}$ and length of $200 \mathrm{~nm} .{ }^{1}$ In order to maintain the thermodynamic stability, DTCs self-assembled into secondary structure of body-cube-center (BBC) lattices which rendered dimension in $200 \times 200 \times 200 \mathrm{~nm}^{3}$. After BBC lattices interacted with electrode surface, non-ideal optical isotropic BPLC re-combined into elliptical structures (a)

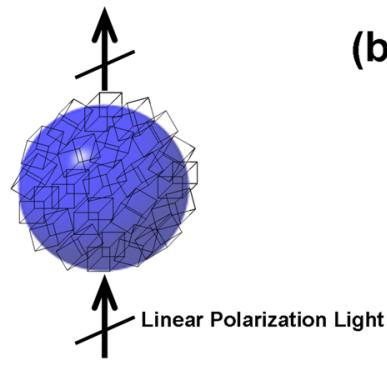

(b)

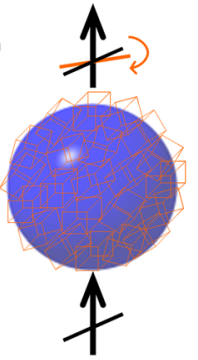

(c)

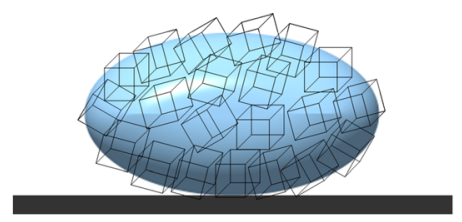

Transparent-Conductive Electrode

Blue Phase Liquid Crystal

Aligned BPLC BPLC Lattice

(d)

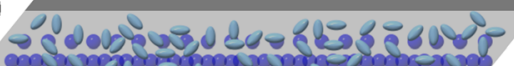

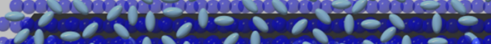
$\sin 28$

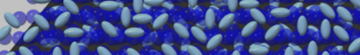

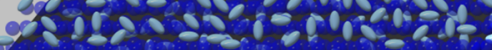

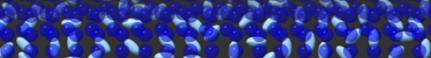

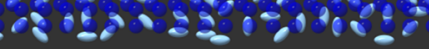

(e)

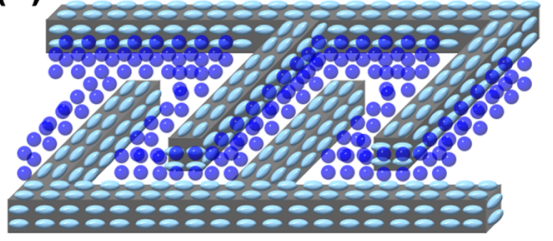

FIG. 2. Conceptual diagram of BPLC self-assembly and alignment: (a) Ideal optical isotropy, (b) non-ideal optical isotropy, (c) self-assembly of BPLC on electrode surface, (d) random distributed in VFS cell, and (e) directional alignment along electrode axis in IPS cell. 
TABLE I. Dark state measurement by colorimeter.

\begin{tabular}{llcl}
\hline \hline & \multicolumn{3}{c}{ Dark state (nit) } \\
\hline Samples & $\theta=0^{\circ}$ & $\theta=45^{\circ}$ & $\theta=90^{\circ}$ \\
JC-BP01M & 0.3565 & 0.5308 & 0.3141 \\
JC-BP06N & 0.1867 & 0.3391 & 0.1973 \\
\hline \hline
\end{tabular}

(i.e., tertiary structures, Figure 2(c)) due to TNLC anchoring force and aspect-ratio effect of nanostructures. ${ }^{15-18}$ Initially, the interaction between BPLC and electrode was raised by TNLC anchoring force. Subsequently, the anchoring was enhanced by aspect-ratio effect due to nanostructure of BBC lattice. Consequently, tertiary structures of BPLC resulted in light leakage, especially in electrode edge. According to dark state result of IPS cell (Figure 1(d)), the curve revealed Gauss distribution which was consistent with the selfassembly of elliptical structures.

Consideration of cell-structure effect, alignment of BPLC showed different behavior in VFS and IPS cell. Comparison of VFS and IPS, BPLC randomly distributed on top and bottom electrode surfaces of VFS (Figure 2(d)), while aligned along with electrode axis of IPS cell (Figure 2(e)). Consequently, VFS revealed more distributed area than IPS and dark state resulted in three-time larger than that of IPS $\left(3.33 \times 10^{-2} \%\right.$ in VFS and $1.07-1.20 \times 10^{-2} \%$ in IPS). VFS revealed no significant change at different $\theta$, because BPLC randomly distributed on electrode surface. In contrary, BPLC directionally aligned on electrode axis of IPS cell and rendered phase retardation along electrode axis. Phase retardation along electrode axis can be greatly reduced by adjusting electrode axis parallel to polarizer or analyzer and revealed small dark state at $\theta=0^{\circ}$ and $45^{\circ}$.

In order to double confirm result of dark state, measurement and BPLC materials were changed. Dark state was obtained by nit index measurement (luminance colorimeter system). ${ }^{19}$ Commercial BPLC of JC-BP01M $(\Delta \varepsilon=94)$ and JC-BP06N ( $\Delta \varepsilon=491)$ were obtained from JNC Corporation. Consequently, nit index (Table I) was consistent with the result of laser-detector system (Figure 1). Both JC-BP01M and JC-BP06N revealed large nit index at $\theta=45^{\circ}$ and small nit index at $\theta=0^{\circ}$ and $90^{\circ}$.

The light leakage of IPS cell can be directly observed by polarized optical microscope (POM) and the images showed large different at $\theta=0^{\circ}-90^{\circ}$. However, POM images were obtained from the same IPS cell. As showed in Figure 3, images of $\theta=0^{\circ}$ and $90^{\circ}$ revealed slightly light leakage (Figures 3(a)-3(d)), while image of $\theta=45^{\circ}$ revealed severe light leakage due to non-ideal optical isotropic effect (Figure 3(e)). In large magnitude of POM image, light leakage was located on the edge of electrode (Figure 3(f)). Consequently, POM image was consistent with laser-detector system and nit index measurement.

In conclusion, BPLC was regarded as optical isotropy for three decades; however, we found it should be non-ideal optical isotropy due to optical rotatory power and selfalignment. BPLC not only revealed primary structure of double-twist cylinder and secondary structure of lattice but also rendered tertiary structure of self-alignment on electrode surface. Furthermore, light leakage was cell dependence and

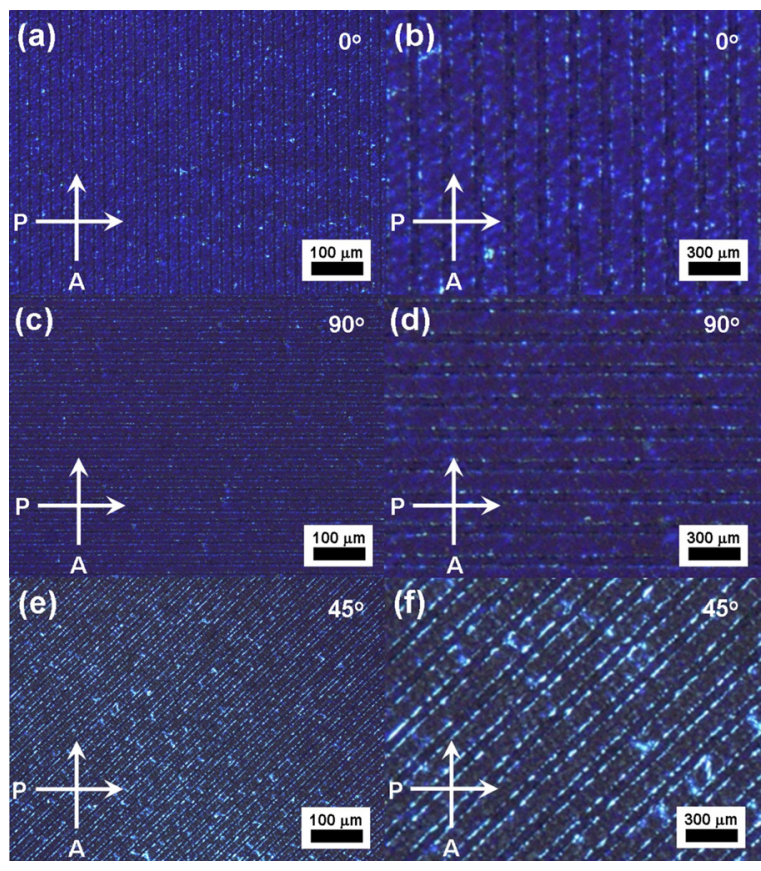

FIG. 3. Images of polarized-optical microscope obtained from the same IPS cell at $\theta=0^{\circ}(\mathrm{a}, \mathrm{b}), 45^{\circ}(\mathrm{e}, \mathrm{f})$, and $90^{\circ}(\mathrm{c}, \mathrm{d})$.

showed different manner. For example, BPLC revealed selfassembly and alignment along with electrode axis in IPS cell and light leakage was observed along with electrode axis. However, BPLC showed randomly distributed in VFS cell and light leakage revealed three-time larger than IPS. We believe this finding of non-ideal optical isotropic BPLC will impact academic researches and industrial applications.

${ }^{1}$ P. P. Crooker, Chirality in Liquid Crystals (Springer, New York, USA, 2001). ${ }^{2}$ C. Kittel, Introduction to Solid State Physics (Wiley, New York, USA, 1996). ${ }^{3}$ K. M. Chen, S. Gauza, H. Xianyu, and S. T. Wu, J. Display Technol. 6, 49 (2010).

${ }^{4}$ Y. Chen, J. Yan, J. Sun, S. T. Wu, X. Liang, S. H. Liu, P. J. Hsieh, K. L. Cheng, and J. W. Shiu, Appl. Phys. Lett. 99, 201105 (2011).

${ }^{5}$ H. Kikuchi, M. Yokota, Y. Hisakado, H. Yang, and T. Kajiyama, Nature Mater. 1, 64 (2002).

${ }^{6}$ Y. Chen, D. Xu, S. T. Wu, S. Yamamoto, and Y. Haseba, Appl. Phys. Lett. 102, 141116 (2013).

${ }^{7}$ Y. Haseba, S. Yamamoto, K. Sago, A. Takata, and H. Tobata, SID Int. Symp. Dig. Tech. Pap. 44, 254 (2013).

${ }^{8}$ H. C. Cheng, J. Yan, T. Ishinabe, and S. T. Wu, Appl. Phys. Lett. 98, 261102 (2011).

${ }^{9}$ Y. F. Lan, C. Y. Tsai, J. K. Lu, and N. Sugiura, Polymer 54, 1876 (2013).

${ }^{10}$ C. D. Tu, C. L. Lin, J. Yan, Y. Chen, P. C. Lai, and S. T. Wu, J. Disp. Technol. 9, 3 (2013).

${ }^{11}$ C. Y. Tsai, T. J. Tseng, L. Y. Wang, F. C. Yu, Y. F. Lan, P. J. Huang, S. Y. Lin, K. M. Chen, B. S. Tseng, C. W. Kuo, C. H. Lin, J. K. Lu, and N. Sugiura, SID Int. Symp. Dig. Tech. Pap. 44, 182 (2013).

${ }^{12}$ H. Lee, H. J. Park, O. J. Kwon, S. J. Yun, J. H. Park, S. Hong, and S. T. Shin, SID Int. Symp. Dig. Tech. Pap. 42, 121 (2011).

${ }^{13}$ Y. Hirakata, D. Kubota, A. Yamashita, T. Ishitani, T. Nishi, H. Miyake, H. Miyairi, J. Koyama, S. Yamazaki, T. Cho, and M. Sakakura, J. Soc. Inf. Disp. 20, 38 (2012).

${ }^{14}$ Y. Liu, Y. F. Lan, H. Zhang, R. Zhu, D. Xu, C. Y. Tsai, J. K. Lu, N. Sugiura, Y. C. Lin, and S. T. Wu, Appl. Phys. Lett. 102, 131102 (2013).

${ }^{15}$ Y. F. Lan, R. H. Lee, and J. J. Lin, J. Phys. Chem. B 114, 1897 (2010).

${ }^{16}$ Y. F. Lan and J. J. Lin, J. Phys. Chem. A 113, 8654 (2009).

${ }^{17}$ Y. F. Lan, B. Z. Hsieh, H. C. Lin, Y. A. Su, Y. N. Chan, and J. J. Lin, Langmuir 26, 10572 (2010).

${ }^{18}$ Y. F. Lan and S. C. Cheng, Appl. Phys. Lett. 100, 153109 (2012).

${ }^{19}$ Y. F. Lan, C. Y. Tsai, L. Y. Wang, P. J. Ku, T. H. Huang, C. Y. Liu, and N. Sugiura, Appl. Phys. Lett. 100, 171902 (2012). 